EPJ Web of Conferences 92,02122 (2015)

DOI: $10.1051 /$ epjconf/ 20159202122

C Owned by the authors, published by EDP Sciences, 2015

\title{
Application of gamma densitometry and statistical signal analysis to gas phase velocity measurements in pipeline hydrotransport
}

\author{
Marcin Zych ${ }^{1, a}$, Robert Hanus ${ }^{2}$, Leszek Petryka ${ }^{1}$, Dariusz Świsulski ${ }^{3}$, Anna Strzępowicz ${ }^{1}$ and Piotr Zych ${ }^{4}$ \\ ${ }^{I}$ AGH University of Science and Technology, Krakow 30-059, Poland \\ ${ }^{2}$ Rzeszow University of Technology, Rzeszow 35-959, Poland \\ ${ }^{3}$ Gdansk University of Technology, Gdansk 80-233, Poland \\ ${ }^{4}$ Warsaw University of Technology, Warszawa 00-661, Poland
}

\begin{abstract}
The work presents selected methods of signal analysis used in the processing of data obtained from radiometric probes. The used data came from an exemplary study of a two-phase liquid-gas flow at the laboratory installation. In such rigs many possible transport types may be observed, i.e. slug, plug and bubble flow, and each of them gives different signal-to-noise ratio of recorded data. Therefore, available radiometric methods of gas phase velocity measurements give diverse accuracies. Authors consider several improvements of data acquisition and processing which increase possibility of the flow type recognition and higher accuracy of the gas phase velocity estimation.
\end{abstract}

\section{Introduction}

A multiphase flow is dominant in natural environment and industry transport. In particular, in such branches as chemical, petrochemical and power plants a liquid-gas flow is widely found. Since those technologies require a control of flow characteristics, it is necessary to construct equipment which allows measurement of such parameters as velocity, flow rate, concentration of gas phase, etc. Among many flowmeters used in such tasks are, for example: ultrasound, Particle Image Velocimetry, Coriolis one, and numerous kind of tomography (capacitive, inductive, resistive, X-ray), or radiometric methods applied by the authors [1-7]. Most of these methods combine advanced mathematical signal processing, similar to those widely used in medicine, geophysics, etc. [8-10].

Due to current development of computing such mathematical techniques, as the cross-correlation (CCF) of recorded signals, or their deconvolution, cross-spectral density function (CSDF), and combined methods are offered [11-17]. Less popular method of signal analysis includes cross-correlation with the Hilbert Transform [18, 19], differential methods [13, 16, 20] and methods based on the conditional averaging of the signals [21-23].

The article presents an arbitrary selected procedure of gas phase velocity determination in a pipeline transportation of water-air mixture.

\section{Base of the gamma absorption method and measurement conditions}

The principle of the gamma-ray absorption measurement, used in a flow examination is shown in figure 1. The sealed ${ }^{241} \mathrm{Am}$ radiation sources of $3.7 \mathrm{GBq}$ activity (2), emit beams of gamma rays with an energy of $59.5 \mathrm{keV}$ through the slots of collimators (1). A beam shaped in this way (7) passes through the pipeline (5) with a flowing compound. Consequently part of the gamma rays may be absorbed by a transported medium. The rest of photons reach the scintillation probes (4), providing $I_{x}(t)$ and $I_{y}(t)$ signals (figure 2), which are subsequently sampled with time $\Delta t=1 \mathrm{~ms}$. Thus origin stochastic digital functions $x(n)$ and $y(n)$, where $n$ is specified:

$$
n=t / \Delta t \text {. }
$$

Figure 1 shows the measurement geometry: size slots of collimators, distances between radioactive sources and detectors, and diameter of the pipeline.

The research installation for liquid-gas flow investigation was constructed at the Laboratory of Industrial Radiometry, Faculty of Physics and Applied Computer Science, AGH - University of Science and Technology in Krakow. View of a part of the installation, is presented in figure 3. More detail description of the testing rig may be found in papers $[15,24-26]$. In the paper an exemplary simulation of air-water mixtures flow in the three regimes, shown in figure 4 was evaluated [24].

\footnotetext{
${ }^{\mathrm{a}}$ Corresponding author: zych@geol.agh.edu.pl
} 


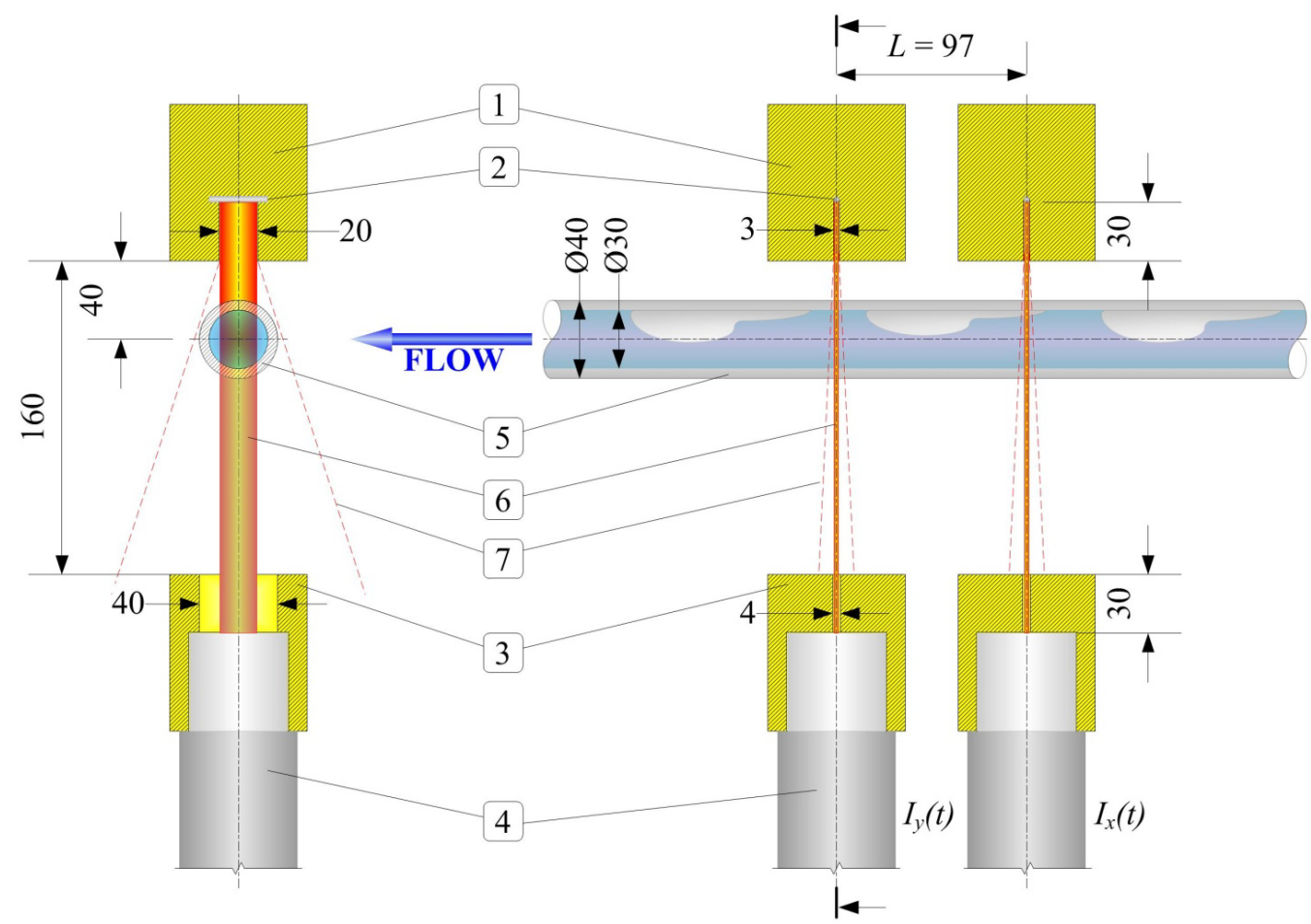

Figure 1. The $\gamma$-ray absorption set: 1 - collimator, $2-{ }^{241} \mathrm{Am}$ source, 3 - collimator of detector, 4 - scintillation probe, 5 - pipeline, 6 - main $\gamma$-ray beam, 7 - dispersed $\gamma$-radiation. All dimensions are given in $\mathrm{mm}$.
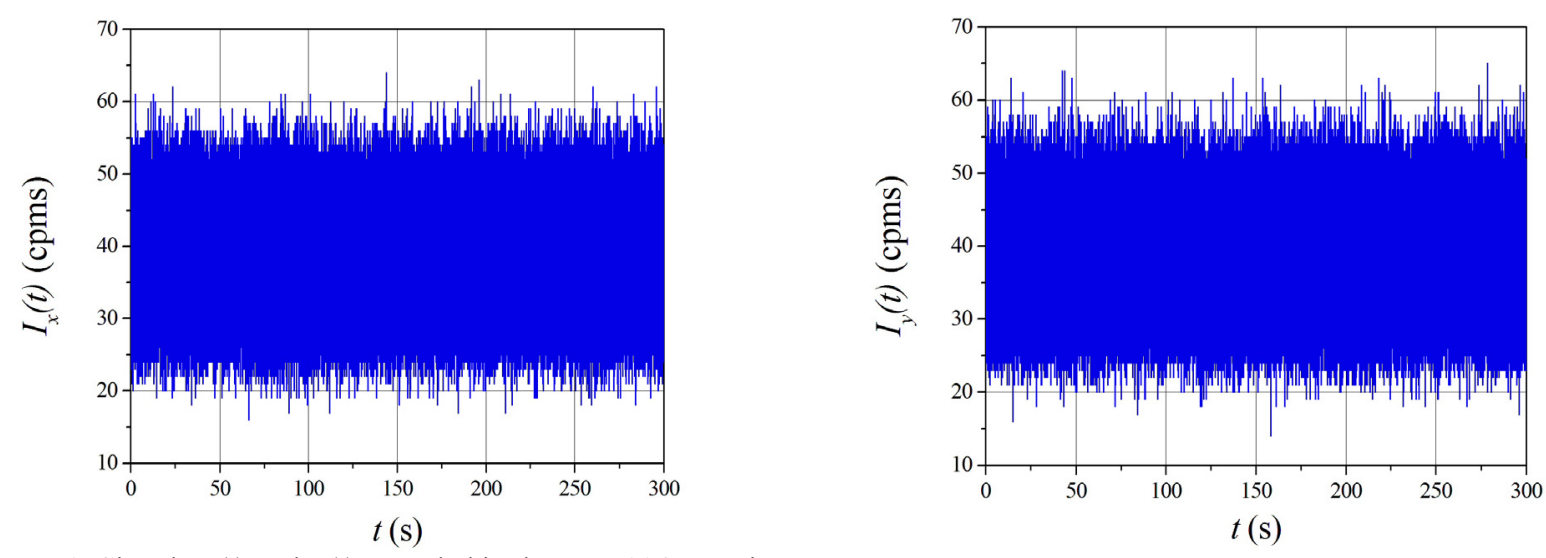

Figure 2. Signals $I_{x}(t)$ and $I_{y}(t)$ recorded in the BUB004 experiment.



Figure 3. View of a part of the laboratory installation.

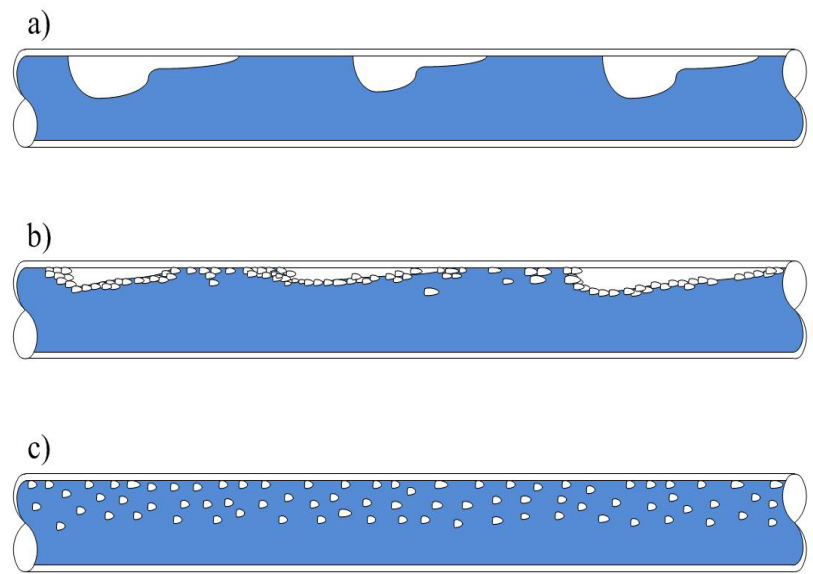

Figure 4. The selected flow patterns: a) plug flow, b) slug flow, c) bubble flow. 


\section{Mathematical methods of signal analysis}

The conducted measurements reveal significant influence of gas phase transportation form and concentration on the recorded signal-to-noise ratio (SNR). Therefore each type of the gas transportation was separately analyzed.

In the paper evaluation of the gas phase velocity base on determination of the transportation time delay and following methods were consider:

- classic cross-correlation function,

- deconvolution (RTD),

- cross-spectral density function.

All calculations below were performed by the "Convolution" software designed under direction of the authors [15].

\subsection{Cross-correlation method}

In case of digital signals, the cross-correlation function is calculated from the formula $[11,12,14]$ :

$$
R_{x y}(\tau)=\frac{1}{N} \sum_{n=0}^{N-1} x(n) \cdot y(n+\tau),
$$

where: $N$ - number of samples, equal to $300000, \tau$ - time delay.

Wherein the transportation time delay estimator is defined as:

$$
\hat{\tau}_{0}=\arg \left\{\max R_{x y}(\tau)\right\}=\arg \left\{R_{x y}\left(\tau_{0}\right)\right\} .
$$

In result of the high noise contribution in the $x(n)$ and $y(n)$ signals, their ergodicity is not fulfilled. In the paper a filtration of these signals was applied as a remedy. It was realized by determination of the cutoff frequency for recorded signals based on their frequency representation $[7,14,15]$.

As a result the autospectral density function of the individual signals (ASDF), or module cross-spectral density function $(|\mathrm{CSDF}|)$ by decomposition signal using the discrete Fourier transform (DFT) may be properly calculated. The CSDF is defined by the relation [12, 24 27]:

$$
G_{x y}(f)=\sum_{\tau=-\infty}^{\infty} R_{x y}(\tau) \cdot e^{-j 2 \pi \cdot f \cdot \tau}
$$

where: $f$ - frequency.

The periodogram $|\mathrm{CSDF}|$, for an exemplary the BUB004 measurement is shown in figure 5, where the red frame indicated the selected frequency band.

The cross-correlation function, by filtration of the recorded signals, shows figure $6 \mathrm{~d}$, e, $\mathrm{f}$.

\subsection{Deconvolution method}

The deconvolution calculation is based on the wellknown mathematical operator. In the case of digital signals it will be determined by summing the products of the input signal $x(n)$ and the response function $h(\tau)$ according to $[28,29]$ :

$$
y(n)=x(n) * h(n)=\sum_{\tau=-\infty}^{\infty} x(n-\tau) \cdot h(\tau) .
$$

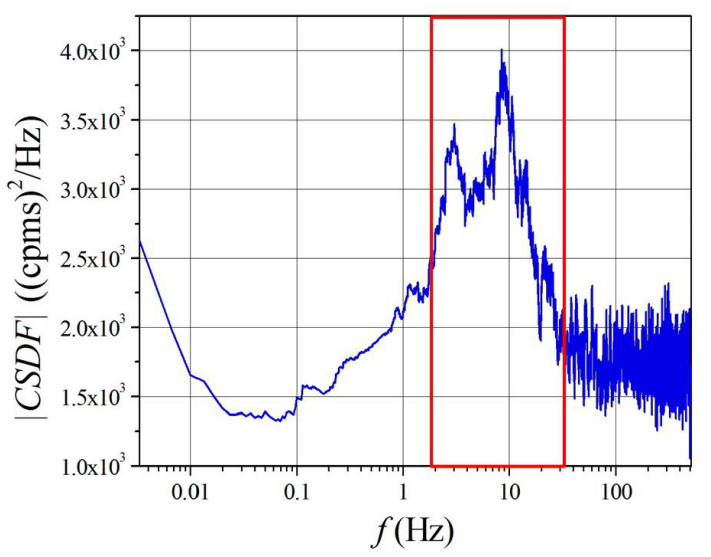

Figure 5. Module of the cross-spectral density function for BUB004 run. Red frame marked the selected frequency band.

The $h(\tau)$ function is interpreted as the residence time distribution RTD of medium in the measuring section [29]. For example, in this case it is the averaged time of air transportation between the probes. Due to simplicity of calculations, the transition time of gas phase was determined in the frequency domain by applying the Discrete Fourier Transform. In this method the time delay $\tau_{0}$ is estimated by position of the maximum function $h(\tau)$. Figures $6 \mathrm{~g}, \mathrm{~h}$, i, illustrate the function $h(\tau)$ for the exemplary data.

\subsection{Phase method}

For the analysis of $x(n)$ and $y(n)$ signals in the frequency domain the phase of the cross spectral density $G_{x y}(f)$ is also associated with time delay $\tau_{0}$ by the following formula:

$$
\Phi_{x y}(f)=\operatorname{arctg}\left\{\frac{\operatorname{Im}\left[\underline{G}_{x y}(f)\right]}{\operatorname{Re}\left[\underline{G}_{x y}(f)\right]}\right\}=2 \pi \cdot f \cdot \tau_{0},
$$

The course of CSDF phase allows determining the $\tau_{0}$ for a specific frequency range $[30,31]$. In this method, the selected range of $\Phi_{x y}(f)$ linear approximation was applied. For the CSDF phase estimation the strings of the Welch procedure for discrete signals was used. Samples collected in this way were divided into $N_{d}$ segments, each of $N$ length.

Through application of the DFT to CSDF, it is possible to obtain the $f_{k}$ discrete frequency values $(k=0$, $1, \ldots(N-1) / 2)$ with the following resolution in the frequency domain:

$$
\Delta f=1 /(N \cdot \Delta t)
$$

The use of the above procedure for the arctan function calculation causes discontinuities in the CSDF phase and creates a necessity of so-called unwrapping phase application. 
BUB006

a)



d)

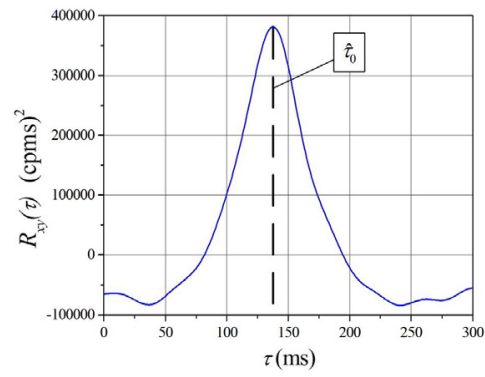

g)

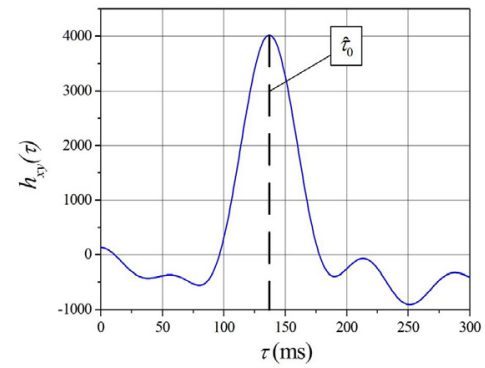

j)



BUB004

b)



e)

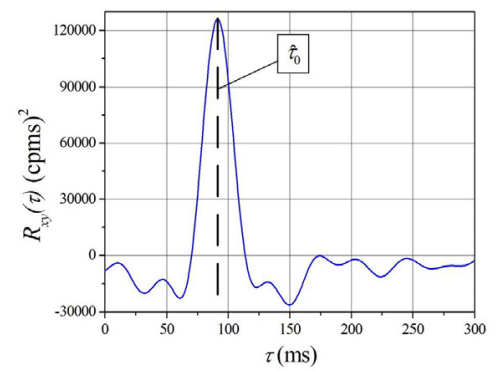

h)



k)

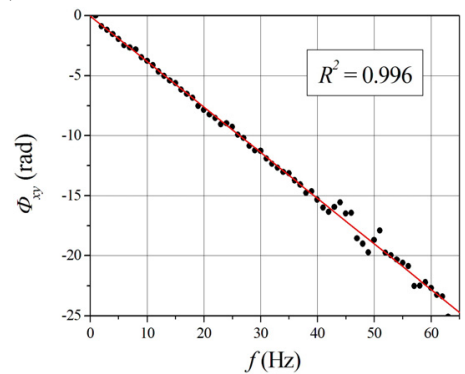

BUB001

c)

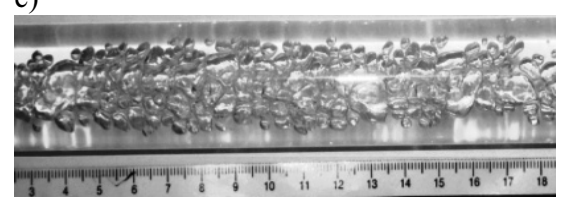

f)

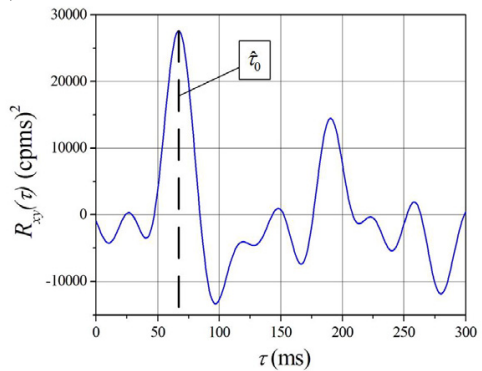

i)

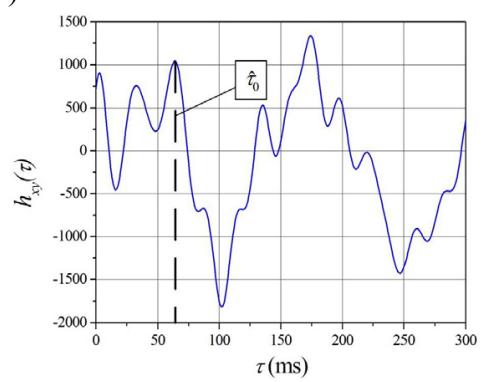

1)

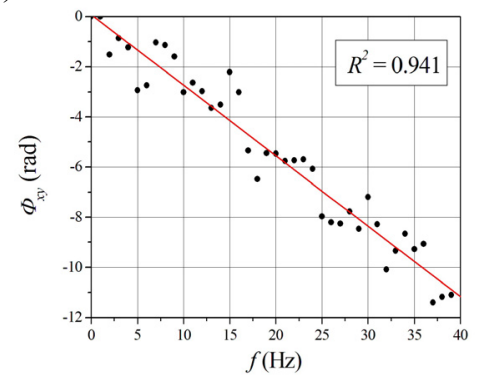

Figure 6. (a, b, c) picture of the flow, (d, e, f) CCF after filtration, (g, h, i) deconvolution, (j, k, l) CSDF phase. Run BUB006 - plug flow, $v_{W}=0.90 \mathrm{~m} / \mathrm{s}$; run BUB004 - slug flow, $v_{W}=1.36 \mathrm{~m} / \mathrm{s}$; run BUB001 - bubble flow, $v_{W}=1.92 \mathrm{~m} / \mathrm{s}$; $v_{W}$ - velocity of water obtain from ultrasonic flowmeter Uniflow 990. Red line marked the best linear fit.

As a result the transport delay time may be estimated by an averaging phase $\widetilde{\Phi}_{x y}\left(f_{k}\right)$ from the relation [ibid.]:

$$
\tau_{0}=-\frac{1}{2 \pi}\left[\sum_{k=1}^{l} f_{k} \cdot \widetilde{\Phi}_{x y}\left(f_{k}\right) / \sum_{k=1}^{l} f_{k}^{2}\right],
$$

where: $l$ - the number of the phase and frequency values of the CSDF included in the regression line calculation. In the figures $6 \mathrm{j}, \mathrm{k}, 1$ the selected fragments of $\widetilde{\Phi}_{x y}\left(f_{k}\right)$ analysis are shown.

\section{The interpretation of results}

The estimated transportation time delay was obtained by fitting a normal distribution to the main peak of the CCF and RTD. Hence, $u_{A}\left(\hat{\tau}_{0}\right)$ is uncertainty of the time delay designation defined as [32]:

$$
u_{A}\left(\hat{\tau}_{0}\right)=\sigma / \sqrt{m},
$$

where: $\sigma$ - the standard deviation of the fitted Gaussian distribution, $m$ - number of the fitted normal distribution points [13, 14, 25].

Knowing the measuring distance $L$ (as shown in figure $1, L=97 \mathrm{~mm}$ ), one can calculate the average velocity of gas phase in the mixture, from the relationship:

$$
v_{G}=L / \hat{\tau}_{0} .
$$

The uncertainty of the measurement section $L$ estimation is of the type $B$, relating directly to the 
measuring gauge deployed as well as accuracy of collimators dimensions, $u_{B}(L)=0.1 \mathrm{~mm}$.

Considering the $v_{G}$ determination, the indirectly used estimation of accuracy of the law of propagation of uncertainty was used [ibid.]:

$$
u_{C}\left(v_{G}\right)=\sqrt{\left[\frac{\partial v_{G}}{\partial L} \cdot u_{B}(L)\right]^{2}+\left[\frac{\partial v_{G}}{\partial \tau_{0}} \cdot u_{A}\left(\hat{\tau}_{0}\right)\right]^{2}} .
$$

The values of the estimated time delay, velocity, and their uncertainties are summarized in tables 1 and 2 .

Table 1. The values of the transportation time delay and the uncertainty.

\begin{tabular}{|c|c|c|c|c|c|c|}
\multirow{2}{*}{ Run } & \multicolumn{2}{|c|}{ BUB006 } & \multicolumn{2}{c|}{ BUB004 } & \multicolumn{2}{c|}{ BUB001 } \\
\cline { 2 - 7 } Method & $\begin{array}{c}\tau_{0} \\
\mathrm{~ms}\end{array}$ & $\begin{array}{c}u_{A}\left(t_{0}\right) \\
\mathrm{ms}\end{array}$ & $\begin{array}{c}t_{0} \\
\mathrm{~ms}\end{array}$ & $\begin{array}{c}u_{A}\left(t_{0}\right) \\
\mathrm{ms}\end{array}$ & $\begin{array}{c}t_{0} \\
\mathrm{~ms}\end{array}$ & $\begin{array}{c}u_{A}\left(t_{0}\right) \\
\mathrm{ms}\end{array}$ \\
\hline CCF & 136.6 & 2.1 & 91.0 & 1.5 & 66.3 & 1.4 \\
\hline RTD & 136.4 & 2.0 & 90.8 & 1.1 & 63.1 & 1.0 \\
\hline CSDF & 136.6 & 0.4 & 91.5 & 0.2 & 66.4 & 1.4 \\
\hline
\end{tabular}

Table 2. The values of average air velocity and the uncertainty.

\begin{tabular}{|c|c|c|c|c|c|c|}
\multirow{2}{*}{ Run } & \multicolumn{2}{|c|}{ BUB006 } & \multicolumn{2}{c|}{ BUB004 } & \multicolumn{2}{c|}{ BUB001 } \\
\cline { 2 - 7 } Method & $\begin{array}{c}v_{G} \\
\mathrm{~m} / \mathrm{s}\end{array}$ & $\begin{array}{c}u_{C}\left(v_{G}\right) \\
\mathrm{m} / \mathrm{s}\end{array}$ & $\begin{array}{c}v_{G} \\
\mathrm{~m} / \mathrm{s}\end{array}$ & $\begin{array}{c}u_{C}\left(v_{G}\right) \\
\mathrm{m} / \mathrm{s}\end{array}$ & $\begin{array}{c}v_{G} \\
\mathrm{~m} / \mathrm{s}\end{array}$ & $\begin{array}{c}u_{C}\left(v_{G}\right) \\
\mathrm{m} / \mathrm{s}\end{array}$ \\
\hline $\mathrm{CCF}$ & 0.710 & 0.011 & 1.066 & 0.017 & 1.463 & 0.032 \\
\hline $\mathrm{RTD}$ & 0.711 & 0.011 & 1.069 & 0.013 & 1.536 & 0.025 \\
\hline $\mathrm{CSDF}$ & 0.710 & 0.002 & 1.060 & 0.003 & 1.460 & 0.031 \\
\hline
\end{tabular}

Analyzing these results, it can be concluded, that the lowest uncertainty is characterized by the CSDF method. However, with increase in the share of noise in recorded signals, the square of the correlation coefficient lowers, which means the inferior fit of a straight line to the data (red line figure $6 \mathrm{j}, \mathrm{k}, 1$ ). Thus the uncertainty of air velocity estimation for run BUB001 by all methods is comparable. Also, when compare values of the RTD uncertainty one can see, that for the CCF it is the lowest.

Authors consider, that the cross-correlation function is the most universal method for $\tau_{0}$ determination. It should be also noted, that for as small noise contribution as in the run BUB006 signal, all methods of data analysis give a very similar results.

\section{Conclusions}

The use of the gamma ray absorption method for measuring the flow velocity of air in the gas-liquid compound, transported via the horizontal pipeline is perspective. This task was achieved by averaging the delay time of the gas phase transport determination, and for recorded data processing including the crosscorrelation function, deconvolution, and phase of cross spectral density.

Moreover, the paper brings comparison of presented methods and their influence on uncertainty of results in case of different noise presence in recorded signals. However, for data with a high signal-to-noise ratio like in the run BUB006 all provided methods give very similar results. In all other cases it was realized, that the most trusty is the analysis using the CCF, and proper filtration of signals provided by such sensors as nuclear probes.

\section{References}

1. G. Falcone, G.F. Hewitt, C. Alimonti, Multiphase flow metering: principles and applications (Elsevier Amsterdam, 2009)

2. M. Bottin, et al, Int. J. Multiphas. Flow 60, 161-179 (2014)

3. T. Xue, L. Qu, Z. Cao, T. Zhang, Flow Meas. Instrum. 27, 29-36 (2012)

4. M.R. Rzasa, Nucl. Eng. Des. 239, 699-707 (2009)

5. G.A. Johansen, P. Jackson, Radioisotope gauges for industrial process measurements (John Wiley, New York 2004)

6. V. Mosorov, Meas. Sci. Technol. 17, 753-760 (2006)

7. L. Petryka, R. Hanus, M. Zych, Prz. Elektrotech. 88 (1b), 185-188 (2012)

8. P. Augustyniak, Physiol. Meas. 24 (3), 753-767 (2003)

9. B. Buttkus, Spectral analysis and filter theory in applied geophysics (Springer-Verlag, Berlin 2000)

10. J. Jarzyna, M. Bała, Ann. Soc. Geol. Pol. 83 (2), 133-147 (2013)

11. S.H. Jung, et al, Appl. Rad. Isotop. 67, 1254-1258 (2009)

12. J.S. Bendat, A.G. Piersol, Random data - analysis and measurement procedures (John Wiley, New York, 2010)

13. R. Hanus, M. Zych, L. Petryka, JPCS 530012042 (2014)

14. M. Zych, et al., Flow Meas. Instrum. 35, 11-15 (2014)

15. L. Petryka, et al, Prz. Elektrotech. 86 (5), 24-29 (2010)

16. R. Hanus, L. Petryka, M. Zych, Flow Meas. Instrum. 40, 58-63 (2014)

17. R. Hanus, Prz. Elektrotech. 84 (12), 301-303 (2008)

18. J.S. Bendat, The Hilbert Transform and applications to correlation measurements Brüel\&Kjær BT000811 (Naerum, Denmark, 1985)

19. R. Hanus, Prz. Elektrotech. 88 (10b), 39-41 (2012)

20. G. Jacovitti, G. Scarano, IEEE Trans. Sign. Proc 41, 525-533 (1993)

21. R. Hanus, Prz. Elektrotech. 86 (6), 232-235 (2010) 
22. A. Kowalczyk et al, Metrol. Meas. Syst. 18, 335342 (2011)

23. R. Hanus, Syst. Anal. Mod. Sim. 40, 239-244 (2001)

24. M. Zych, R. Hanus, L. Petryka, MATEC web conf. (to be published in 2014)

25. R. Hanus, et al, Proc. 2012 IEEE Mediterranean Electrotechnical Conference MELECON 2012, 144147 (2012)

26. L. Petryka, M. Zych, R. Hanus, MAaM 54 (12), 863-865 (2008)

27. J.H. McClellan, R.W. Schafer, M. A. Yoder, Signal processing first (Pearson Prentice Hall, Upper Saddle River, New Jersey 2003)
28. A.V. Oppenheim, R.W. Schafer, J.R. Buck, Discrete-Time Signal Processing (Pearson Prentice Hall, Upper Saddle River, New Jersey 1999)

29. L. Petryka, Proc. 12th international conference on Transport \& sedimentation of solid particles. 12th international symposium on Freight pipelines 1, 5974 (2004)

30. A.G. Piersol, IEEE Trans. Acoust. Speech Sign Proc. 29, 471-477 (1981)

31. R. Hanus, L. Petryka, M. Zych, MAaM 58 (3), 236239 (2012)

32. Guide to the expression of uncertainly in measurement (International Organization for Standardization, 1995) 